\title{
Two-loop evaluation of large Wilson loops with overlap fermions: the b-quark mass shift, and the quark-antiquark potential
}

\author{
Andreas Athenodorou* \\ Department of Physics, University of Cyprus, Nicosia CY-1678, Cyprus \\ E-mail: ph00aalducy.ac.cy \\ Haralambos Panagopoulos \\ Department of Physics, University of Cyprus, Nicosia CY-1678, Cyprus \\ E-mail: haris@ucy.ac.cy
}

\begin{abstract}
We compute, to two loops in pertubation theory, the fermionic contribution to rectangular $R \times T$ Wilson loops, for different values of $R$ and $T$.

We use the overlap fermionic action. We also employ the clover action, for comparison with existing results in the literature. In the limit $R, T \rightarrow \infty$ our results lead to the shift in the b-quark mass. We also evaluate the perturbative static potential as $T \rightarrow \infty$.
\end{abstract}

XXIIIrd International Symposium on Lattice Field Theory

25-30 July 2005

Trinity College, Dublin, Ireland

\footnotetext{
* Speaker.
} 


\section{Introduction}

In this work, we compute the perturbative value of large Wilson loops up to two loops, using the clover ( $\mathrm{SW}$ ) and overlap fermions. Using the perturbative values of Wilson loops of infinite length, we evaluate the shift of the b-quark mass. The perturbative values of Wilson loops of infinite time extent lead us to the evaluation of the static potential. For the case of clover fermions, we also compare our results with established results.

The calculation of Wilson loops in lattice perturbation theory is useful in a number of ways: a) It leads to the prediction of a strong coupling constant $a_{\overline{M S}}\left(m_{Z}\right)$ from low energy hadronic phenomenology by means of non-perturbative lattice simulations (for a list of relevant references, see our longer write-up [1]). b) It is employed in the context of mean field improvement programmes of the lattice action and operators. c) In the limit of infinite time separation, $T \rightarrow \infty$, Wilson loops give access to the perturbative quark-antiquark potential. Furthermore in the limit of large distances, $R \rightarrow \infty$, the self energy of static sources can be obtained from the potential, enabling the calculation of $\overline{m_{b}}\left(\overline{m_{b}}\right)$ from non-perturbative simulations of heavy-light mesons in the static limit [2].

\section{Calculation of Wilson loops}

The Wilson loop $W$, around a closed curve $C$, is the expectation value of the path ordered product of the gauge links along $C . W(R, T)$ denotes a rectangular Wilson loop with dimensions $R \times T$. There are two Feynman diagrams involving fermions, contributing to $W(R, T)$ at two loops, as shown in Fig. 1.

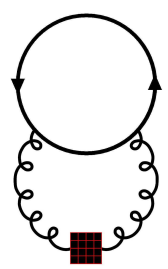

1

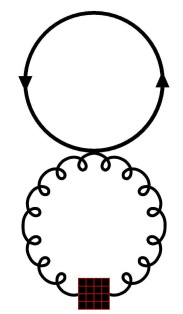

2

Fig.1: Two-loop fermionic diagrams contributing to $W(R, T)$.

The grid-like square stands for the 2-point vertex of $W(R, T)$, whose expression is:

$$
\begin{aligned}
& W(R, T) \rightarrow-\frac{g^{2}}{24} \sum_{\mu, v} \int \frac{d^{4} k d^{4} k^{\prime}}{(2 \pi)^{4}} A_{\mu}^{a}(k) A_{v}^{b}\left(k^{\prime}\right) \delta^{a b} \delta\left(k+k^{\prime}\right)\left[2 \delta_{\mu, v} \mathrm{~S}\left(k_{\mu}, R\right) \sum_{\rho} \sin ^{2}\left(k_{\rho} a T / 2\right)\right. \\
& \left.+2 \delta_{\mu, v} \mathrm{~S}\left(k_{\mu}, T\right) \sum_{\rho} \sin ^{2}\left(k_{\rho} a R / 2\right)-4 \mathrm{~S}\left(k_{\mu}, R\right) \mathrm{S}\left(k_{v}, T\right) \sin \left(k_{\mu} a / 2\right) \sin \left(k_{v} a / 2\right)\right]
\end{aligned}
$$

where: $\mathrm{S}\left(k_{\mu}, R\right) \equiv \sin ^{2}\left(R k_{\mu} a / 2\right) / \sin ^{2}\left(k_{\mu} a / 2\right)$.

The involved algebra of the lattice perturbation theory was carried out using our computer package in Mathematica. The value of each diagram is computed numerically for a sequence of finite lattice sizes. Their values have been summed, and then extrapolated to infinite lattice size. 


\section{Calculation with Clover Fermions}

The fermionic part of the action contains an additional (clover) term, parameterized by a coefficient, $c_{S W}$, which is a free parameter in the present work; $c_{S W}$ is normally tuned in a way as to minimize $\mathscr{O}(a)$ effects. The perturbative expansion of the Wilson loop is given by the expression:

$$
W(C) / N=1-W_{L O} g^{2}-\left(W_{N L O}-\frac{\left(N^{2}-1\right)}{N} N_{f} X\right) g^{4},
$$

where: $W_{L O}$ and $W_{N L O}$ are the pure gauge contributions with the Wilson gauge action, which can be found in Ref. [3], 田, and:

$$
X=X_{W}+X_{S W}^{a} c_{S W}+X_{S W}^{b} c_{S W}^{2} .
$$

We have computed the values of $X_{W}, X_{S W}^{a}$, and $X_{S W}^{b}$. We compare our results with those of Ref. [П] (only $X_{W}$ ) , and Ref. [3] (for $m=0$ ). Our results for $X_{W}, X_{S W}^{a}$, and $X_{S W}^{b}$ as a function of mass for square loops, are shown in Figs. 2-4.

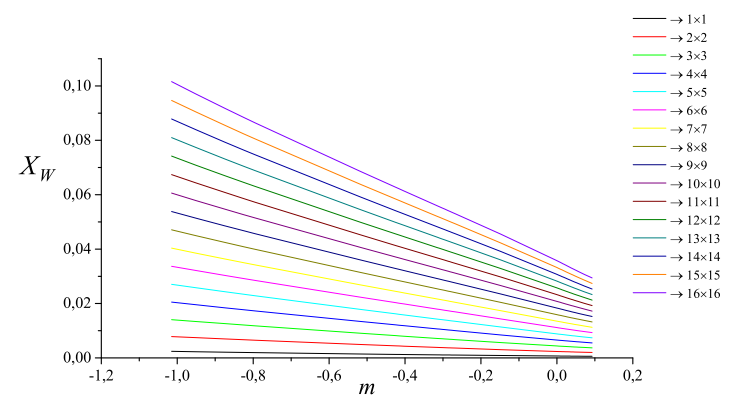

Fig.2: $X_{W}$ for loops $L \times L$. Top line: $L=16$, bottom line: $L=1$.

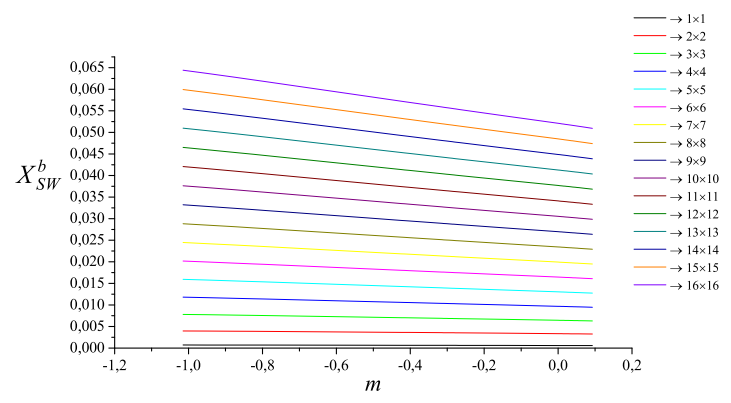

Fig.4: $X_{S W}^{b}$ for loops $L \times L$. Top line: $L=16$, bottom line: $L=1$.

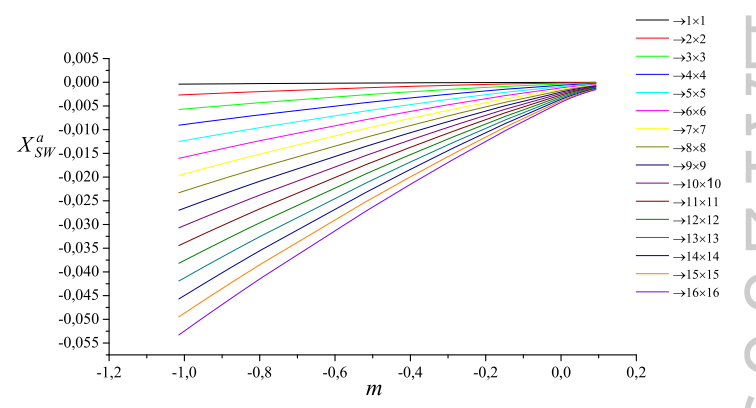

Fig.3: $X_{S W}^{a}$ for loops $L \times L$. Top line: $L=1$, bottom line: $L=16$.

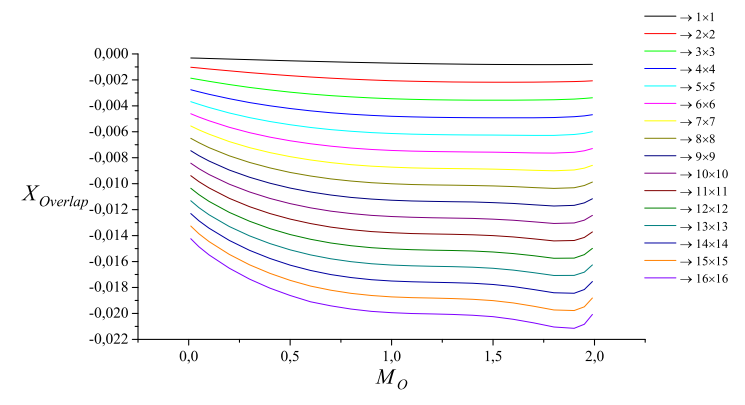

Fig.5: $X_{\text {Overlap }}$ for loops $L \times L$. Top line: $L=1$, bottom line: $L=16$.

\footnotetext{
${ }^{1}$ In order to compare with Ref. [4], we deduce their values of $X_{W}$ from the data presented there and we estimate the errors stemming from that data.
} 


\section{Calculation with Overlap Fermions}

The fermionic action now reads

$$
S_{F}=\sum_{f} \sum_{x, y} \bar{\psi}_{x}^{f} D_{\mathrm{N}}(x, y) \psi_{y}^{f} .
$$

with: $D_{\mathrm{N}}=M_{O}\left[1+X\left(X^{\dagger} X\right)^{-1 / 2}\right]$, and: $X=D_{\mathrm{W}}-M_{O}$; the index $f$ runs over $N_{f}$ flavours. Here, $D_{\mathrm{W}}$ is the massless Wilson-Dirac operator with $r=1$, and $M_{O}$ is a free parameter whose value must be in the range $0<M_{O}<2$, in order to guarantee the correct pole structure of $D_{\mathrm{N}}$.

Details on the propagator and vertices of $S_{F}$ can be found in our Ref. [5] and references therein. The perturbative expansion of the Wilson loop is given by the expression:

$$
W(C) / N=1-W_{L O} g^{2}-\left(W_{N L O}-\frac{\left(N^{2}-1\right)}{N} N_{f} X_{\text {Overlap }}\right) g^{4},
$$

where $X_{\text {Overlap }}$ are the values we compute. Fig. 5 shows $X_{\text {Overlap }}$ as a function of $M_{O}$ for square Wilson loops. Tables of our numerical values are presented in our Ref. [1].

\section{Calculation of the b-quark mass shift}

In perturbation theory, the expectation value of large Wilson loops decreases exponentially with the perimeter of the loops:

$$
\langle W(R, T)\rangle \sim \exp (-2 \delta m(R+T)) .
$$

Following Ref. [2], the perturbative expansion for $\langle W(R, T)\rangle$ is:

$$
\langle W(R, T)\rangle=1-g^{2} W_{2}(R, T)-g^{4} W_{4}(R, T)+\mathscr{O}\left(g^{6}\right) .
$$

Using the expectation value of $W(R, T)$, we obtain the perturbative expansion for $\delta m$ :

$$
\delta m=\frac{1}{2(R+T)}\left[g^{2} W_{2}(R, T)+g^{4}\left(W_{4}(R, T)+\frac{1}{2} W_{2}^{2}(R, T)\right)\right]
$$

$W_{2}(R, T)$ involves only gluons and: $W_{4}(R, T)=W_{4}^{g}(R, T)+W_{4}^{f}(R, T)$, where $W_{4}^{g}(R, T)$ is the contribution in the pure gauge theory and $W_{4}^{f}(R, T)$ is the fermionic contribution.

To evaluate the effect of fermions on the mass shift, we must examine their contribution in the limit as $R, T \rightarrow \infty$. To this end, we note that our expression assumes the generic form (modulo terms which will not contribute in this limit):

$$
\int \frac{d^{4} p}{(2 \pi)^{4}} \sin ^{2}\left(p_{v} T / 2\right) \sin ^{2}\left(p_{\mu} R / 2\right)\left(\frac{1}{\sin ^{2}\left(p_{v} / 2\right)}+\frac{1}{\sin ^{2}\left(p_{\mu} / 2\right)}\right) \frac{1}{\left(\hat{p}^{2}\right)^{2}} G(p),
$$

where $\hat{p}^{2}=4 \sum_{\rho} \sin ^{2}\left(p_{\rho} / 2\right)$ and $G(p) \sim p^{2}$. As $R, T \rightarrow \infty$, the above expression becomes: 


$$
\frac{1}{2}(R+T) \int \frac{d^{3} \bar{p}}{(2 \pi)^{3}} \frac{1}{\left(\hat{\bar{p}}^{2}\right)^{2}} G(\bar{p})
$$

where: $\bar{p}=\left(p_{1}, p_{2}, p_{3}, 0\right)$ (for $\mu=4$ or $\left.v=4\right)$.

The fermionic contribution takes the form:

$$
W_{4}^{f}(R, T)=\left(N^{2}-1\right) N_{f}(R+T) V
$$

where $V \equiv V_{W}+V_{S W}^{a} c_{S W}+V_{S W}^{b} c_{S W}^{2}$ for clover fermions, and $V \equiv V_{\text {Overlap }}$ for overlap fermions. The values of $V_{W}, V_{S W}^{a}, V_{S W}^{b}$ and $V_{\text {Overlap }}$ have been calculated in the present work. Figs. 6 and 7 show their values as a function of bare mass for clover fermions and of $M_{O}$ for overlap fermions:

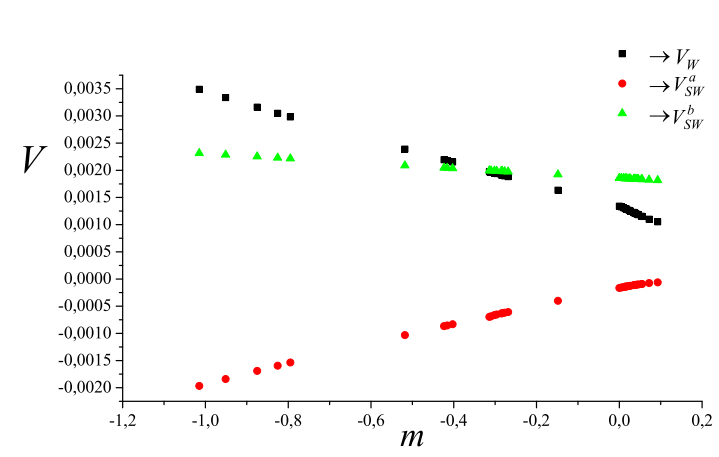

Fig.6: $V_{W}, V_{S W}^{a}$ and $V_{S W}^{b}$ as a function of $m$.

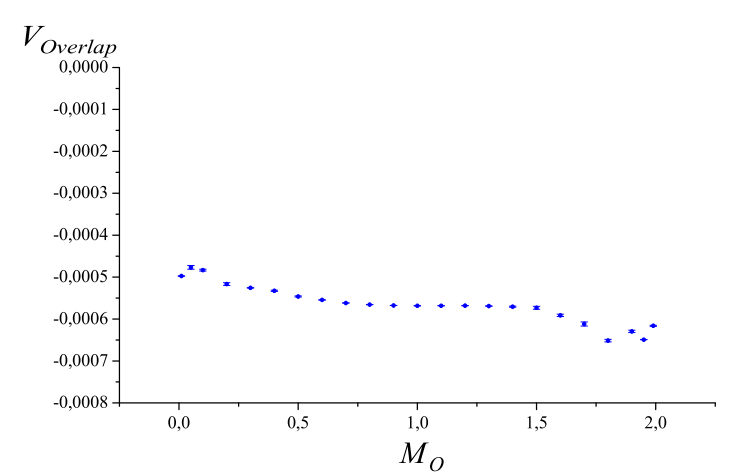

Fig.7: $V_{\text {Overlap }}$ as a function of $M_{O}$.

At one-loop order the b-quark mass shift (for $N=3$ ) is given by:

$$
a \delta m \simeq 0.16849 g^{2}+\mathscr{O}\left(g^{4}\right),
$$

Using our results arrive at the two-loop expression for $\delta m$. We list below some examples: The general form of $\delta m$ is $\left(\alpha_{0}=g^{2} / 4 \pi\right)$ :

$$
a \delta m \simeq 2.1173 \alpha_{0}+\left[11.152-\frac{(4 \pi)^{2}\left(N^{2}-1\right) N_{f}}{2 N} V\right] \alpha_{0}^{2}+\mathscr{O}\left(\alpha_{0}^{3}\right)
$$

For clover fermions, setting $m=0.0$, we find:

$$
V=0.00134096(5)-0.0001641(1) c_{S W}+0.00185871(2) c_{S W}^{2}
$$

These numbers agree with the result given in Ref. [2], for the case $c_{S W}=0$, within the precision presented there. For overlap fermions, setting $M_{O}=1.4$, our result is:

$$
V_{\text {Overlap }}=0.000571(1)
$$




\section{Calculation of the Perturbative Static Potential}

The static potential is given by the expression:

$$
a V(R a)=-\lim _{T \rightarrow \infty} \frac{d \ln W(R, T)}{d T}=V_{1}(R) g^{2}+V_{2}(R) g^{4}+\ldots,
$$

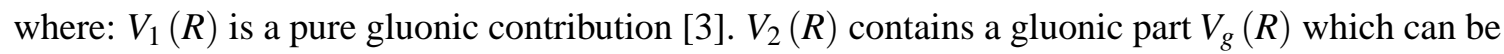
found in Ref. [3] and a fermionic part $F(R)$ :

$$
V_{2}(R)=V_{g}(R)-\frac{\left(N^{2}-1\right)}{N} N_{f} F(R)
$$

We compute $F(R)$ for clover and overlap fermions. For clover:

$$
F(R)=F_{\text {Clover }}(R)=F_{W}(R)+F_{S W}^{a}(R) c_{S W}+F_{S W}^{b}(R) c_{S W}^{2},
$$

and for overlap: $F(R)=F_{\text {Overlap }}(R)$. The values of $F_{W}(R), F_{S W}^{a}(R), F_{S W}^{b}(R)$ and $F_{\text {Overlap }}(R)$ for specific mass values, can be found in the Tables of our Ref. [1]. We also present them in Fig. 8 (for $c_{S W}=1.3$ ) and Fig. 9, as a function of $R$.

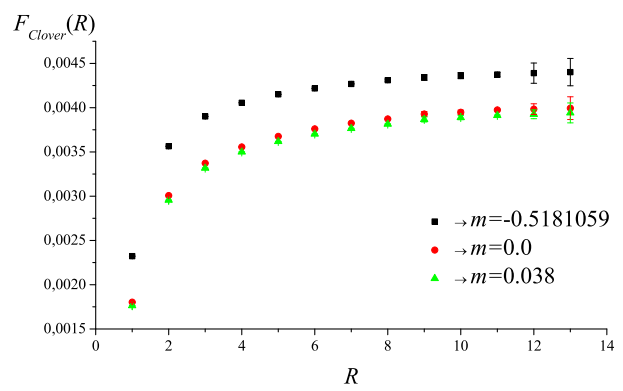

Fig.8: $F_{\text {Clover }}$ as a function of $R\left(c_{S W}=1.3\right)$.

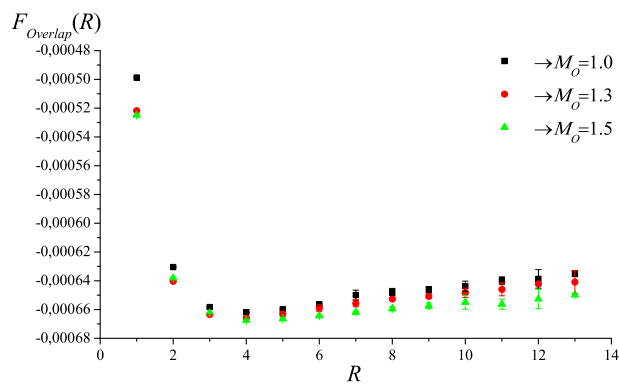

Fig.9: $F_{\text {Overlap }}$ as a function of $R$.

\section{References}

[1] A. Athenodorou and H. Panagopoulos, Large Wilson loops with overlap and clover fermions: Two-loop evaluation of the b-quark mass shift, and the quark-antiquark potential [hep-lat/0509039].

[2] G. Martinelli and C. T. Sachrajda, Computation of the b-quark Mass with Perturbative Matching at the Next-to-Next-to-Leading Order, Nucl. Phys. B559 (1999) 429 [hep-lat/9812001].

[3] G. Bali and P. Boyle, Perturbative Wilson loops with massive sea quarks on the lattice [hep-lat/0210033].

[4] F. Di Renzo and L. Scorzato, Numerical Stochastic Perturbation Theory for full QCD, JHEP 0410 (2004) 073 [hep-lat/ 0410010$].$

[5] A. Athenodorou and H. Panagopoulos, The Lattice Free Energy with Overlap Fermions: A Two-Loop Result, Phys. Rev. D70 (2004) 077507 [hep-lat / 0408020 ]. 